\title{
Identification of HLA-A*02-B*46 haplotype allele variant in Guangdong Han populations on the basis of PCR-SBT Fu Xiong ${ }^{2}$, Lulu Xiao*1, Min $\mathrm{Luo}^{1}$ and Fei Huang ${ }^{1}$
}

Address: ${ }^{1}$ The Department of Tissue Typing Center, Nanfang Hospital, Southern Medical University, Guangzhou 510515, Guangdong, PR China and ${ }^{2}$ The Department of Medical Genetics, School of Basic Medical Sciences, Southern Medical University, Southern Medical University, Guangzhou 510515, Guangdong, PR China

Email: Fu Xiong - xiongfu@smu.edu.cn; Lulu Xiao* - xiaolulu_54@yahoo.com.cn; Min Luo - bearbear_cn@163.com; Fei Huang - gzhuangfei@21cn.com

* Corresponding author

Published: 7 April 2009

BMC Research Notes 2009, 2:55 doi:10.1 I86/1756-0500-2-55
Received: 26 July 2008

Accepted: 7 April 2009

This article is available from: http://www.biomedcentral.com/I756-0500/2/55

(c) 2009 Xiao et al; licensee BioMed Central Ltd.

This is an Open Access article distributed under the terms of the Creative Commons Attribution License (http://creativecommons.org/licenses/by/2.0), which permits unrestricted use, distribution, and reproduction in any medium, provided the original work is properly cited.

\begin{abstract}
Background: The HLA-A*02-B*46 haplotype is one of most frequent haplotypes among Guangdong Han populations. To explore the characteristics of the HLA-A*02-B*46 haplotype in Guangdong Han populations, the genetic polymorphism of HLA-A*02-B*46 haplotype was analysed by PCR-SBT in our study.

Findings: Among 88 samples with the homozygotes for HLA-A*02-B*46 in the low resolution, 4 different alleles for $A * 02(A * 020 I, A * 0203, A * 0206, A * 0207)$ and I allele for $B * 46(B * 460 I)$ were identified by PCR-SBT. Among them, the $A^{*} 0207$ allele was the predominant allele. Inversely, among the samples with HLA-A*2-B*46(-), six alleles were detected for $A * 02(A * 020101, A * 0203$, $A * 0205, A^{*} 0206$ and $\left.A * 0207\right)$, and the $A^{*} 020 \mathrm{I}$ allele was predominant. On the other hand, the HLA-A*02-B*46 haplotype presented moderate heterozygosis (32.95\%). In addition, the linkage with DRBI was analysed in HLA-A*2-B*46 haplotype, and there existed 10 alleles with DRBI. With the low resolution for DRBI, the other I0 DRBI alleles all linked with the HLA-A*02-B*46 haplotype except for DRBI*0I, DRBI*I0, and DRBI*I7. Moreover, we found eight alleles of DRBI in the HLA-A*0207-B*460I haplotype.

Conclusion: The polymorphism distribution of the $A * 02$ allele between the HLA-A*02-B*46 and HLA-A*02-B*46(-) haplotypes among the Guangdong Han populations provides useful information for research on unrelated hematopoietic stem cell transplantation (UHSCT), anthropology, and disease association for populations with the HLA-A*02-B*46 haplotype.
\end{abstract}

\section{Background}

HLA-A*02 is the most common allele in humans [1,2], and is the most heterogeneous HLA-A specificity, with 97 subtypes [3]. HLA-B*46 is the most common allele in East Asian populations and occurs at varying frequencies in different populations, including $14 \%$ among the Thais,
$13.2 \%$ among the Vietnamese, $4.4 \%$ among the Japanese, and $10 \%$ among the Chinese [4]. The HLA-A*02-B*46 haplotype is one of most common haplotypes in Chinese populations, especially the Guangdong Han population. HLA-A*02-B*46 presents significant linkage disequilibrium, which shows its genetic dominance. Moreover, it is 
shown that the HLA-A*02-B*46 allele is positively related to certain diseases, such as nasopharyngeal carcinoma, which has a high incidence in Guangdong province $[5,6]$.

The intent of this study was to explore the polymorphism of the HLA-A*02-B*46 haplotype homozygote and its distribution characteristics in the Guangdong Han population, and also to establish a bone marrow bank with HLA$A * 02-B * 46$ haplotype to improve the effect of bone marrow transplantation and decrease the occurrence of GVHD. Therefore, we have analysed the genetic polymorphism of HLA-A* $02-B^{*} 46$ in Guangdong Han population by polymerase chain reaction-sequence-based typing (PCR-SBT) and report our findings in this paper.

\section{Methods \\ Subjects}

For all subjects, genomic DNA was isolated from white cells in $5 \mathrm{ml}$ peripheral blood using the DNA Extraction Kit (Qiagen) according to the manufacturer's specifications. Primary genotyping was carried out by PCR-RSSO (Dynal Biotech Ltd, Invitrogen) on 8191 blood samples from unrelated bone marrow donors from the Guangdong Han population. Of the 8191 donors, 88 [mean age (35.5 \pm 7.8 ) years, range $18 \sim 55$ years] that were typed as HLA-A*02-B* 46 positive were used in the present investigation. They were all ethnic Han, and their families have lived in Guangdong Province for at least three generations. As a control, 150 samples with $\mathrm{A}^{*} 02$ and without the HLA-B*46 allele were selected in research. These selected samples were homozygotes for HLA-A*02-B*46 in the low resolution (PCR-RSSO). The local ethics committee reviewed the study and informed consent was obtained from all the participants.

\section{HLA-A*02-B*46 genotyping}

HLA typing was performed by PCR-SBT analysis as previously described after amplifying the second exon of HLA$A$, the third exon of HLA-B, and the second exon of HLADRB1 [7]. High resolution typing of the HLA-A*02, B*46, and DRB1 alleles was performed by PCR-SBT using the ABI 3100 with the software Matchtool. The primers used for PCR-amplification and sequencing of HLA-A, B*46, DRB1 were listed in table 1 and 2, respectively.

\section{Statistical analysis}

Relative frequencies of the HLA-A*2-B*46 allele were calculated by direct counting. $\chi^{2}$ analysis was used to assess statistically significant differences between the HLA-A*02$\mathrm{B}^{*} 46$ and HLA-A*02-B*46(-) haplotype, Pc is obtained by $P$ multiplying the number of alleles at each locus. Statistical analyses were conducted with an SPSS software program.

\section{Results \\ Allele distribution of the $A * 02$ and $B * 46$ genes in the HLA- A*02-B*46 haplotype}

In the 88 samples, $176 \mathrm{~A}^{*} 02-\mathrm{B} * 46$ haplotypes were detected. Only four $A^{*} 2$ alleles including $A^{*} 0201$, $A^{*} 0203, A^{*} 0206, A^{*} 0207$ were detected in 176 HLA$A^{*} 02-B^{*} 46$ haplotypes. Of the four $A^{*} 2$ alleles, $A^{*} 0207$ allele was the predominant allele with a frequency of $77.3 \%$, followed by A*0201, A*0203, and A*0206 with respective frequencies of $14.2 \%, 4.0 \%$ and $4.5 \%$ (Table $3)$. In the control group, six $A^{*} 02$ alleles were detected, including $A^{*} 0201, A^{*} 0203, A^{*} 0205, A^{*} 0206, A^{*} 0207$ and $A^{*} 0210$; the $A^{*} 0201$ allele was predominant with a frequency of $55.3 \%$ (Table 3 ). The $A^{*} 0205$ and $A^{*} 0210$ alleles were not detected in the HLA-A*02-B*46 haplotype. Additionally, only one $B^{*} 46$ alleles with $B^{*} 4601$ was observed in $176 \mathrm{~A}^{*} 02-\mathrm{B}^{*} 46$ haplotypes. There existed significant difference with the frequencies of $A^{*} 2$ subtypes between HLA-A*02-B*46 haplotype and HLA-A*02B*46(-) haplotype.

\section{Heterozygosis of $A * 02-B * 46$ haplotype in the Guangdong Han population}

HLA-A*02-B*46 haplotype presents homozygosis at low resolution. However, at high resolution, HLA-A*02-B*46 haplotype possess moderate heterozygosis and the ratio is $32.95 \%$. Although $\mathrm{A}^{*} 02$ presents a polymorphism in HLA-A*02-B*46 haplotypes, the ratio of heterozygosis with $A^{*} 02$ is low. The major homozygous alleles in the HLA-A*02-B*46 haplotypes of the Guangdong Han population are HLA-A*0207-B*4601 and $A^{*} 0207-B^{*} 4601$, and the major heterozygotes are HLA-A*0201-B*4601 and HLA-A*0207-B*4601 (Table 4).

\section{The diversity of the DRBI allele linked to the HLA-A*02- B*46 haplotype}

From 55 homozygotes for HLA-A*02-B*46 haplotype, the polymorphism of the DRB1gene was analyzed. We found 10 DRB1 alleles, including DRB1*04, DRB1*07, DRB1*08, DRB1*09, DRB1*11, DRB1*12, DRB1*13, $\mathrm{DRB} 1 * 14, \mathrm{DRB} 1 * 15, \mathrm{DRB} 1 * 15$ and DRB1*16 (Table 5). Among the 10 DRB1 alleles, the DRB1*09 is the most common allele in the Guangdong Han population. A polymorphism of the DBR1 gene was detected in the HLA$A^{*} 0207-B * 4601$ haplotype that existed as 8 DRB1 alleles, including $\mathrm{DRB} 1 * 04, \mathrm{DRB} 1 * 08, \mathrm{DRB} 1 * 09, \mathrm{DRB} 1 * 11$, DRB1*12, DRB1*14, DRB1*15, DRB1*16 (Table 5). Among the $8 \mathrm{DRB} 1$ alleles, the $\mathrm{DRB} 1{ }^{*} 09$ is also the most common allele in the HLA-A*0207-B*4601 haplotype.

\section{Discussion}

The HLA-A*02-B*46 haplotype is one of most frequent haplotypes found in Southern Chinese Han populations, 
Table I: Sequence specific oligonucleotide used for PCR-amplification of HLA-A, B*46, DRB I

\begin{tabular}{|c|c|c|c|c|}
\hline Gene Names & Primer Names & Nucleotide sequence(5'-3') & $\mathrm{Tm}^{\circ}$ & Position \\
\hline \multirow[t]{2}{*}{ HLA-A } & AintronIF & 5'-CTC TG(C/T) GGG GAG AAG CAA-3' & 58 & $4864-488 I^{\mathrm{a}}$ \\
\hline & Aex5mod $\mathrm{R}$ & 5'-CCA GCA A(G/T)G ATG CCC ACG AT-3' & 62 & $6735-6754^{a}$ \\
\hline \multirow[t]{2}{*}{ HLA-B } & BXI F & 5'-GGG AGG AGC GAG GGG ACC (C/G)CA G-3' & 74 & $5624-5645^{\circ}$ \\
\hline & BINT R & 5'-GGA GGC CAT CCC CGG CGA CCT AT-3' & 78 & $4703-4725^{b}$ \\
\hline \multirow[t]{2}{*}{ HLA-DRBI*0I } & DRBOIF & 5'-TCC CAG TGC CCG CTC CCT-3' & 62 &,$- *$ \\
\hline & DRBOI R & 5'-ACA CAC TCA GAT TCT CCG CTT-3' & 62 &,$- *$ \\
\hline \multirow[t]{2}{*}{ HLA-DRBI*I5, 16} & DRB02 F & 5'-GGT GGG TGC TGT TGA AGG T-3' & 60 &,$- *$ \\
\hline & DRB02 R & 5'-ACA CAC ACA CTC AGA TTC CCA-3' & 62 &,$- *$ \\
\hline \multirow[t]{2}{*}{ HLA-DRBI*03 (exclude03I7), I402, I406, I4I3 } & DRB03 F & 5'-AGC ACT AAG GAA GGG TTC AG-3' & 60 &,$- *$ \\
\hline & DRB03 R & 5'-ACA CAC ACA CTC AGA TTC CCA-3' & 62 &,$- *$ \\
\hline \multirow[t]{2}{*}{ HLA-DRBI*04 } & DRB04 F & 5'-CCT GGG ATC AGA GGT AGA TTTT-3' & 62 &,$- *$ \\
\hline & DRB04 R & 5'-ACA CAC ACA CTC AGA TTC TCC-3' & 62 &,$- *$ \\
\hline \multirow[t]{2}{*}{ HLA-DRBI*07 } & DRB07 F & 5'-CGG CGT CGC TGT CAG TGT T-3' & 62 &,$- *$ \\
\hline & DRB07 R & 5'-TCA GAT TCC CAG CTC GGA GA-3' & 62 &,$- *$ \\
\hline \multirow[t]{2}{*}{ HLA-DRBI*08 } & DRB08 F & 5'-AGC GCA GGC CAG GCT CAA A T-3' & 62 &,$- *$ \\
\hline & DRB08 R & 5'-ACA CAC ACA CTC AGA TTC CCA-3' & 62 &,$- *$ \\
\hline \multirow[t]{2}{*}{ HLA-DRBI*09 } & DRB09 F & 5'-CAG TTA AGG TTC CAG TGC CA-3' & 60 &,$- *$ \\
\hline & DRB09 R & 5'-ACA CAC ACA CTC AGA TTC CCA-3' & 62 &,$- *$ \\
\hline \multirow[t]{2}{*}{ HLA-DRBI*I0 } & DRBIOF & 5'-GGC GTT GCG GGT CGG CG-3' & 62 &,$- *$ \\
\hline & DRBIOR & 5'-ACA CAC AGA GTC AGA TTC CCA-3' & 62 &,$- *$ \\
\hline \multirow[t]{2}{*}{ HLA-DRBI*03(exclude03I7), II, I3, 14} & DRBII.I F & 5'-TGG TGG GCG TTG GGG CG-3' & 60 &,$- *$ \\
\hline & DRBII.I R & 5'-ACA CAC ACA CTC AGA TTC CCA-3' & 62 &,$- *$ \\
\hline \multirow[t]{2}{*}{ HLA-DRBI*II, I3(exclude I3।3), 14} & DRBII.2 F & 5'-AGC ACT AAG GAA GGG TTC AC-3' & 60 & $-*$ \\
\hline & DRBII.2R & 5'-TGT CAC CTC CCC ACA GAG T-3' & 60 &,$- *$ \\
\hline \multirow[t]{2}{*}{ HLA-DRBI*03, II,I3(exclude $|3| 3 \& 03 \mid 7$ ) } & DRBII.3 F & 5'-GTT TTC CCG CCT GGT CCC C-3' & 64 &,$- *$ \\
\hline & DRBII.3 R & 5'-TGT CAC CTC CCC ACA GAG T-3' & 60 &,$- *$ \\
\hline \multirow[t]{2}{*}{ HLA-DRBI*I2 } & $\mathrm{DRB} / 2 \mathrm{~F}$ & 5'-AAC AGG CTG GAG GTA CGG AC-3' & 64 &,$- *$ \\
\hline & $\mathrm{DRB} / 2 \mathrm{R}$ & 5'-ACA CAC ACA CTC AGA TTC CCA-3' & 62 &,$- *$ \\
\hline \multirow[t]{2}{*}{ HLA-DRBI*|30I,| 302} & DRBI3.I F & 5'-GTG GGC GTT GCG GCG GC-3' & 66 &,$- *$ \\
\hline & DRBI3.I R & 5'-ACA CAC ACA CTC AGA TTC CCA-3' & 62 &,$- *$ \\
\hline \multirow[t]{2}{*}{ HLA-DRBI*I4(excludel402, I406, I4I3) } & DRBI4 F & 5'-GTT TTC CCG CCT GGA CCC T-3' & 62 &,$- *$ \\
\hline & DRBI4 R & 5'-ACA CAC ACA CTC AGA TTC CCA-3' & 62 &,$- *$ \\
\hline \multirow[t]{2}{*}{ HLA-DRBI*07 } & DRB07 F & 5'-CGG CGT CGC TGT CAG TGT T-3' & 62 &,$- *$ \\
\hline & DRB07 R & 5'-TCA GAT TCC CAG CTC GGA GA-3' & 62 &,$- *$ \\
\hline \multirow[t]{2}{*}{ HLA-DRBI*08 } & DRB08 F & 5'-AGC GCA GGC CAG GCT CAA A T-3' & 62 &,$- *$ \\
\hline & DRB08 R & 5'-ACA CAC ACA CTC AGA TTC CCA-3' & 62 &,$- *$ \\
\hline \multirow[t]{2}{*}{ HLA-DRBI*09 } & DRB09 F & 5'-CAG TTA AGG TTC CAG TGC CA-3' & 60 &,$- *$ \\
\hline & DRB09 R & 5'-ACA CAC ACA CTC AGA TTC CCA-3' & 62 &,$- *$ \\
\hline \multirow[t]{2}{*}{ HLA-DRBI*10 } & DRBIOF & 5'-GGC GTT GCG GGT CGG CG-3' & 62 &,$- *$ \\
\hline & DRBIOR & 5'-ACA CAC AGA GTC AGA TTC CCA-3' & 62 &,$- *$ \\
\hline
\end{tabular}

a, Numbering of the HLA-A sequence is from the GeneBank accession NT 007952

b, Numbering of the HLA-B sequence is from the GeneBank accession NT 007592

*, Numbering of the HLA-DRBI sequence is from the reference 7 and http://www.ihwg.org

especially in the Guangdong Han population. The HLA$\mathrm{A}^{*} 02-\mathrm{B} * 46$ haplotype has been reported to possibly be related to the genesis of nasopharyngeal carcinoma, the most common cancer in Guangdong province $[5,6]$. By means of linkage analysis of the HLA-A*02-B*46 haplotype, it may be possible to detect NPC genetic susceptibility cases in NPC high risk families. In our study, the HLAA*02, HLA-B*46, HLA-DRB1 alleles and HLA-A*02-B*46 haplotypes in the Guangdong Han population were analysed by high-resolution SBT to study their genetic background. This approach was more efficient than traditional genetic methods, which were based on sequence-specific oligonucleotide probe (SSOP) and sequence-specific primers (SSP).

The HLA-A*02-B*46 haplotype showed high genetic diversity in the Guangdong Han population because of the polymorphism of $A^{*} 02$, although we found only one $\mathrm{B}^{*} 46$ allele with $\mathrm{B} * 4601$ in HLA-A*02-B*46 haplotypes. HLA-A*02 alleles can be classified into two subgroups, including $A^{*} 0201$ and $A^{*} 0205$, by the diversity of the DNA sequence. Almost all populations have $A^{*} 0201$; however, $A^{*} 0203, A^{*} 0206, A^{*} 0207$ and $A^{*} 0210$ have only been found in Oriental populations, while $A^{*} 0205$, 
Table 2: Sequence specific oligonucleotide used for sequencing of HLA-A*02, B*46 and DRB

\begin{tabular}{|c|c|c|c|c|}
\hline Gene Names & Primer Names & Nucleotide sequence $\left(5^{\prime}-3\right.$ ') & $\mathrm{Tm}^{\circ}$ & Position \\
\hline \multirow[t]{2}{*}{ HLA-A Exon 2} & $\mathrm{~A} 5.92 \mathrm{~F}$ & 5'-TCG GGC (A/G)GG TCT CAG CC-3' & 60 & $4934-4950^{\mathrm{a}}$ \\
\hline & AEx2 2R & 5'-CAC TCA CCG GCC TCG CTC TGG-3' & 68 & $5222-5242^{a}$ \\
\hline \multirow[t]{2}{*}{ HLA-A Exon 3} & A5. $103 \mathrm{~F}$ & 5'-GGG CTC GGG GGA C(C/T)G GG-3' & 64 & $5438-5454^{a}$ \\
\hline & A3.4 3R & 5'-GAG GCG CCC CGT GGC-3' & 58 & $5762-5776^{a}$ \\
\hline \multirow[t]{2}{*}{ HLA-B Exon 2} & $\mathrm{BEX} 2 \mathrm{~F}$ & 5'-GGG CGC AGG ACC (T/C)G(A/G) GGA-3' & 58 & $5602-5619 b$ \\
\hline & BEX 2R & 5'-GGT CAC TCA CCG (G/T)CC TCG-3' & 58 & $5272-5289 b$ \\
\hline \multirow[t]{2}{*}{ HLA-B Exon 3} & BEX 3F & 5'-GGG GCC AGG GTC TCA CA-3' & 58 & $5028-5044^{b}$ \\
\hline & BEX 3R & 5'-CCC ACT GCC CCT GGT ACC-3' & 62 & $4744-476 I^{\mathrm{b}}$ \\
\hline \multirow[t]{2}{*}{ HLA-DRBI Exon 2} & $2|M| 3 F$ & 5'-TGT AAA ACG ACG GCC AGT-3' & 54 &,$- *$ \\
\hline & MI3R & 5'-CAG GAA AGA GCT ATG ACC-3' & 54 &,$- *$ \\
\hline
\end{tabular}

a, Numbering of the HLA-A sequence is from the GeneBank accession NT 007952

b, Numbering of the HLA-B sequence is from the GeneBank accession NT 007592

*, Numbering of the HLA-DRBI sequence is from the reference 7 and http://www.ihwg.org

$\mathrm{A}^{*} 0208$ and $\mathrm{A}^{*} 0209$ are known to exist only in white populations. Furthermore, $\mathrm{A}^{*} 0202$ and $\mathrm{A}^{*} 0214$ have been found to exist only in black populations, while $\mathrm{A}^{*} 0211$ are usually found only in Orientals and among South American Indians [3,8-10]. In our research, only four $\mathrm{A}^{*} 02$ alleles were detected in the HLA-A*02-B*46 haplotype and the $\mathrm{A}^{*} 0205$ and $\mathrm{A}^{*} 0210$ alleles were not detected. It may be that $\mathrm{A}^{*} 0205$ and $\mathrm{A}^{*} 0210$ alleles do not exist in the HLA-A*02-B*46 haplotype. On the other hand, we found that the $\mathrm{A}^{*} 0207$ allele was the predominant allele $(77.3 \%)$ in the HLA-A*02-B*46 haplotype. It has been shown that HLA-A*0207-B*4601 is the most common subtype in the HLA-A*02-B*46 haplotype within the Guangdong Han population. However, when compared with the HLA-A*02-B*46(-) haplotype, the polymorphism of the $A^{*} 02$ allele shows a conspicuous diversity in the HLA-A*02-B*46 haplotype. The A*0201 allele was found to be the predominant allele $(55.3 \%)$ in the HLA-A*02-B*46(-) haplotype. Conversely, the $A^{*} 0207$ allele had a measured frequency of only $21.3 \%$. Therefore, the polymorphism distribution of $A^{*} 02$ allele between the HLA-A*02-B*46 and HLA-A*02-B*46(-) haplotypes is significantly different within the ethnic Han

Table 3: Compared with the polymorphism of alleles between HLA-A*02-B*46 haplotype and HLA-A*02-B*46(-) haplotype

\begin{tabular}{cccccc}
\hline$A * 02$ Allele & \multicolumn{2}{l}{ HLA-A*02-B*46 } & \multicolumn{2}{l}{ HLA-A*02-B*46(-) } & Pc \\
\cline { 2 - 5 } & Number & $R F(\%)$ & Number & $\operatorname{RF}(\%)$ & \\
\hline$A^{* 0201}$ & 22 & 12.5 & 83 & $55.3^{*}$ & $\mathrm{Pc}<0.05$ \\
$A^{* 0203}$ & 9 & 5.1 & 9 & 6 & $\mathrm{Pc}>0.05$ \\
$A^{* 0205}$ & 0 & 0 & 5 & $3.3^{*}$ & $\mathrm{Pc}<0.05$ \\
$A^{* 0206}$ & 12 & 6.8 & 18 & $12 *$ & $\mathrm{Pc}<0.05$ \\
$A^{* 0207}$ & 133 & 75.6 & 32 & $21.3^{*}$ & $\mathrm{Pc}<0.05$ \\
$A^{* 0210}$ & 0 & 0 & 3 & $2 *$ & $\mathrm{Pc}<0.05$ \\
Total & 176 & 100 & 150 & 100 & $\mathrm{Pc}<0.05$ \\
\hline
\end{tabular}

$*$ Pc $<0.05$, significantly different compared with HLA-A*02-B*46. population. Most studies have shown that $\mathrm{A}^{*} 0201$ is the prevalent gene in Chinese Han populations and, therefore, is not useful as a genetic marker. However, some investigations have found that at higher resolution, $\mathrm{A}^{*} 0201$ does present diversity between North and South Chinese Han populations which could be regarded as a genetic marker $[11,12]$. In our study, the frequency of $\mathrm{A}^{*} 0201$ presents diversity between different haplotypes, so further investigations of the subtypes are important to determine the frequency of $\mathrm{A}^{*} 0201$.

High-resolution SBT was applied to analyse the alleles of HLA-A*02-B*46 in our study; this technique has shown that the HLA-A*02-B*46 presents heterozygosis to some extent because of the diversity associated with the $\mathrm{A}^{*} 02$ allele. However, the frequency of heterozygosis was lower than the HLA-A*02 haplotype, which has been reported to show $85 \%$ heterozygosis [11]. The cause may be the high proportion of $A^{*} 0207-B^{*} 4601$. The low heterozygosity of HLA-A*02-B*46 makes it easy to look for unrelated donors with HSCT to transplant into patients with the HLA-A*02-B*46 haplotype.

We have found 10 DRB1 alleles in the HLA-A*02-B*46 haplotype. With the low resolution for DRB1, the other DRB1 alleles all exhibited linked behaviour and linked with the HLA-A*02-B*46 haplotype except for DRB*01, $\mathrm{DRB}^{*} 10$, and $\mathrm{DRB}^{*} 17$. However, there were only 8 DRB1 alleles in the HLA-A*0207-B*4601 haplotypes, as

Table 4: The heterozygosis of HLA-A*02-B*46 haplotype

\begin{tabular}{ccccc}
\hline HLA-A*02-B*46 haplotype & $A * 0201$ & $A * 0203$ & $A * 0206$ & $A * 0207$ \\
\hline$A * 0201$ & 2 & 2 & 2 & 14 \\
$A * 0203$ & & 1 & 2 & 3 \\
$A * 0206$ & & & 1 & 6 \\
$A * 0207$ & & & & 55
\end{tabular}


Table 5: The polymorphism of DRB I allele linked by HLA-A*02$B * 46$ haplotype and HLA-A*0207-B*460I haplotype

\begin{tabular}{ccccc}
\hline & \multicolumn{2}{c}{ HLA-A*02-B*46-DRBI } & \multicolumn{2}{l}{ HLA-A*0207-B*460I-DRBI } \\
\cline { 2 - 5 } DRBIAllele & Number & RF(\%) & Number & RF(\%) \\
\hline DRBI*04 & 11 & 6.25 & 2 & 1.82 \\
DRBI*07 & 4 & 2.27 & 0 & 0 \\
DRBI*08 & 12 & 6.82 & 14 & 12.73 \\
DRBI*09 & 114 & 64.78 & 73 & 66.36 \\
DRBI*II & 7 & 3.98 & 5 & 4.55 \\
DRBI*12 & 5 & 2.84 & 5 & 4.55 \\
DRBI*13 & 4 & 2.27 & 0 & 0 \\
DRBI*14 & 10 & 5.68 & 4 & 3.63 \\
DRBI*I5 & 6 & 3.41 & 4 & 3.63 \\
DRBI*16 & 3 & 1.7 & 3 & 2.73 \\
Total & 176 & 100 & 110 & 100 \\
\hline
\end{tabular}

DRB*07 and $\mathrm{DRB}^{*} 13$ were not detected in the HLA$A^{*} 0207-B^{*} 4601$ haplotypes in our study. DRB1*08 has been reported to be a common extended HLA-A*02$\mathrm{B} * 46-\mathrm{DRB} 1$ haplotype among the Japanese and Koreans (24.40\% in frequency) [12]; however, DRB1*09 was the most common allele in the HLA-A*02-B*46 haplotype in South-East Asia [13]. Our results indicate that the DRB1*09 is also the most common allele in the HLA$A * 02-B * 46-D R B 1$ haplotype within the Guangdong Han population, with a frequency of $66.36 \%$

In conclusion, the polymorphism and distribution of HLA-A*02-B*46 haplotypes in the Guangdong Han population shares some genetic characteristics with other populations in other parts of China or other countries. These data provide useful information for unrelated hematopoietic stem cell transplantation (UHSCT), anthropology, and disease association studies.

\section{Abbreviations}

HLA: Human Leukocyte Antigen; RF: relative frequency.

\section{Competing interests}

The authors declare that they have no competing interests.

\section{Authors' contributions}

FX and FH collectively conceived the study, designed the experiment; FX wrote the manuscript; $\mathrm{FH}$ prepared the tables for the manuscript; FH and ML carried out PCR and HLA-A*02-B*46 genotyping. LX contributed to the conception and design of the experiment and were also involved in editing the manuscript. All of the authors have read and approved the final manuscript.

\section{Acknowledgements}

The authors are grateful to the blood donors. This work was supported by Grants from the dean with School of Basic Medical Sciences of Southern Medical University (JC0702).

\section{References}

I. Bodmer JG, Marsh SG, Albert ED, Bodmer WF, Bontrop RE, Dupont B, Erlich HA, Hansen JA, Mach B, Mayr WR, Parham P, Petersdorf EW, Sasazuki T, Schreuder GM, Strominger JL, Svejgaard A, Terasaki PI: Nomenclature for factors of the HLA system. Tissue Antigens 1998, 53:407.

2. Krausa $P$, Browning MJ: HLA-A2 polymorphism and immune functions. Eur J Immunogenet 1996, 23:26I-264.

3. Marsh SG, The WHO Nomenclature Committee for Factors of the HLA System: Nomenclature for factors of the HLA system, update September 2005. Tissue Antigens 2006, 67:94-95.

4. Fleischhauer K, Zino E, Mazzi B, Severini GM, Benazzi E, Bordignon C: HLA-A*02 subtype distribution in Caucasians from northern Italy: identification of A*0220. Tissue Antigens 1996, 48:673-679.

5. Chan SH: Aetiology of nasopharyngeal carcinoma. Ann Acad Med Singapore 1990, 19:201-207.

6. Baoxiang O, Huaye R, Limei L, Jiangbin L: HLA-A2-B46 Haplotype in nasopharyngeal carninoma high risk families. Chinese Medical Genetics 1994, I I: I32-134.

7. Sayer D, Whidborne R, Brestovac B, Trimboli F, Witt C, Christiansen F: HLA-DRB I DNA sequencing based typing: an approach suitable for high throughput typing including unrelated bone marrow registry donors. Tissue Antigens 200I, 57:46-54.

8. Ellis JM, Henson V, Slack R, Ng J, Hartzman RJ, Katovich Hurley C: Frequencies of HLA-A2 Alleles in Five U.S. Population Groups Predominance of $A^{*} 020 I I$ and Identification of HLA-A*023 I. Human Immunology 2000, 6 I:334-340.

9. Shankarkumar U, Prasanavar D, Ghosh K, Mohanty D: HLA A*02 allele frequencies and $B$ haplotype associations in Western Indians. Hum Immunol 2003, 64:562-566.

10. Fleischhauer K, Zino E, Arevalo-Herrera M, Herrera S, Valmori D, Cerottini JC, Benazzi E, Bordignon C, Romero P: Differential expression of HLA-A*02 subtypes in Colombian Blacks and Mestizos. Tissue Antigens 1998, 5 I (2):204-209.

II. Cheng LH, Jin SZ, Gao SQ, Li Z, Zou HY, Wang DM, Wu GG: Difference in HLA-A*02 allele distribution between Han populations in south and north China. Di Yi Jun Yi Da Xue Xue Bao 2005, 25:32I-324.

12. Morishima Y, Kawase T, Malkki M, Petersdorf EW, International Histocompatibility Working Group in Hematopoietic Cell Transplantation Component: Effect of HLA-A2 allele disparity on clinical outcome in hematopoietic cell transplantation from unrelated donors. Tissue Antigens 2007, 69(SuppI I):3I.

13. Park MH, Whang DH, Kang SJ, Han KS: HLA-A*02 allele frequencies and haplotypic associations in Koreans. Tissue Antigens 2000, 55:250-256.

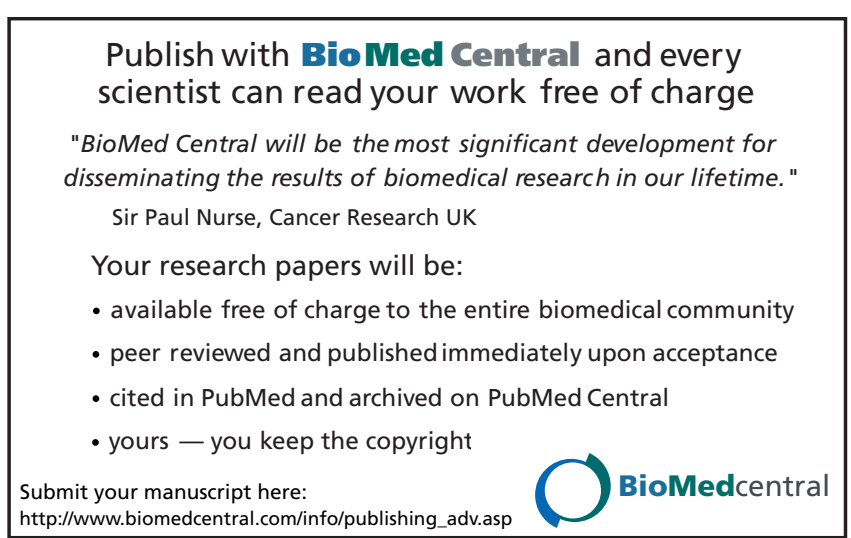

